Cahiers Charlevoix

Études franco-ontariennes
Cahiers Charlevoix Études franco-ontariennes

or Crevenerix of

\title{
Créance populaire et nostalgie du passé : Le Détroit des légendes, de Marie Caroline Watson Hamlin (1884)
}

\section{Michel Gaulin}

Volume 5, 2002

URI : https://id.erudit.org/iderudit/1039352ar

DOI : https://doi.org/10.7202/1039352ar

Aller au sommaire du numéro

\section{Éditeur(s)}

Société Charlevoix

Presses de l’Université d'Ottawa

ISSN

1203-4371 (imprimé)

2371-6878 (numérique)

Découvrir la revue

Citer cet article

Gaulin, M. (2002). Créance populaire et nostalgie du passé : Le Détroit des légendes, de Marie Caroline Watson Hamlin (1884). Cahiers Charlevoix, 5, 141-152. https://doi.org/10.7202/1039352ar
Résumé de l'article

Michel Gaulin porte son regard sur un curieux recueil de la fin du XIX ${ }^{\mathrm{e}}$ siècle, Le Détroit des légendes, un ouvrage publié en anglais par Marie Caroline Watson Hamlin. Il considère d'un point de vue littéraire tant l'édition originale de 1883 que la traduction que Richard Ramsay en a faite en 1991. Bien que les données biographiques sur l'auteur soient incomplètes et les références utilisées pour l'élaboration de l'ouvrage soient vagues et ne donnent guère prise à une confrontation des sources, notre collègue sonde les thèmes légendaires et les allusions historiques des trente et un récits du livre pour définir l'image que l'écrivain donne de la région du Détroit. Il établit encore un parallèle entre le travail de cet écrivain et les activités des artisans du mouvement littéraire de Québec, qui, dans les décennies précédentes, misèrent aussi sur des matériaux populaires du même ordre pour créer leurs oeuvres littéraires. 


\section{CRÉANCE POPULAIRE ET NOSTALGIE DU PASSÉ: LE DÉTROIT DES LÉGENDES, de Marie Caroline Watson Hamlin (1884)}

Michel Gaulin

Département d'études françaises

et College of the Humanities

Université Carleton, Ottawa 


\section{Sommaire}

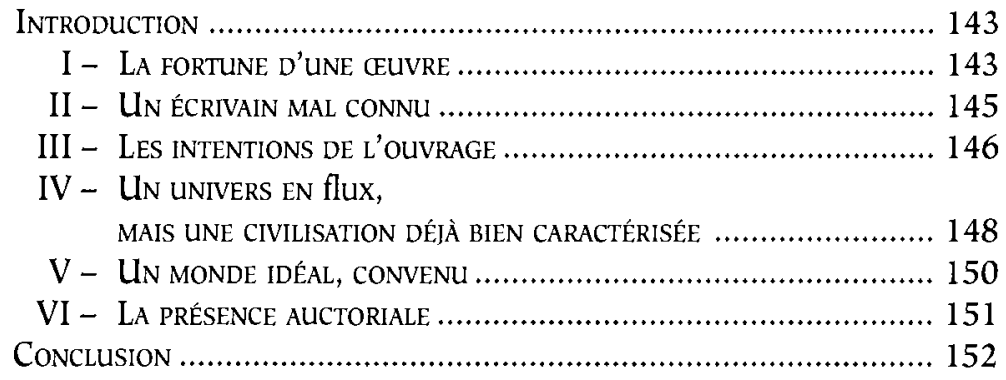


CRÉANCE POPUl.AiRe ET NOSTALGIE DU PASSÉ: LE DÉTROIT DES LÉGENDES, de Marie Caroline Watson Hamlin

\section{(1884)}

\section{INTRODUCTION}

La présente étude se veut un examen principalement littéraire du Détroit des légendes (Legends of Le Détroit), de Marie Caroline Watson Hamlin, œuvre parue d'abord en anglais, en 1884. Aussi rapide qu'il soit, ce bref tour d'horizon permet de constater que le souvenir du passé français du Détroit - de même que celui de la civilisation qu'il véhiculait - était encore bien vivant, vers la fin du XIX siècle, sur les lieux mêmes qui en avaient été le théâtre tout au moins dans l'esprit de quelques individus férus d'histoire et passionnés par les vieilles légendes qui avaient envoûté l'imagination de leurs ancêtres et continuaient d'enchanter la leur.

\section{I - LA FORTUNe d'UNE CEUVRE}

Comme nous l'avons précisé ci-dessus, l'œuvre a d'abord paru en anglais, en 1884, sous le titre Legends of Le Détroit (avec des illustrations d'Isabella Stewart) chez un éditeur 
de Détroit, Thorndike Nourse ${ }^{1}$. Il allait ensuite s'écouler plus d'un siècle avant qu'elle ne connaisse une nouvelle vie. En 1977, la Gale Research Company, également de Détroit, en donnait une réimpression en fac-similé2 et, en 1980, l'Institut canadien de microreproductions historiques la rendait disponible sur microfiches ${ }^{3}$, édition qui est celle que l'on retrouve, de nos jours, dans la plupart des bibliothèques canadiennes.

C'est en 1991, par ailleurs, que l'œuvre paraissait pour la première fois en français, sous le titre Le Détroit des légendes, dans une remarquable traduction annotée de Richard Ramsay, publiée à Sudbury par la Société historique du Nouvel-Ontario ${ }^{4}$. Cette édition avait, sur l'édition originale et ses reproductions en fac-similé, l'avantage de rectifier un certain nombre d'imprécisions d'ordre historique dues à l'auteur et celle, en outre, d'identifier, grâce à cette ressource merveilleuse qu'est le Dictionnaire biographique du Canada, bien des personnages historiques évoqués dans le cours du récit. Enfin, en 2000, la traduction de Richard Ramsay, légèrement révisée, faisait l'objet d'une réédition destinée au commerce, sous le titre Du Crapaud à cheval au Nain rouge. Légendes de Détroit ${ }^{5}$. C'est cette

${ }^{\top}$ Marie Caroline Watson Hamlin, Legends of Le Détroit. Illustrated by Miss Isabella Stewart, Détroit, Thorndike Nourse, 1884 [c 1883], 317 p. D'après les données du catalogue de la Bibliothèque nationale du Canada, une seconde édition aurait paru la même année que la première, soit 1884 , et une troisième l'année suivante, 1885 , toujours chez l'éditeur d'origine. Nous remercions notre collègue Jean-Pierre Pichette, qui possède un exemplaire de la première édition, d'avoir bien voulu nous le confier en dépôt pour nous permettre de poursuivre notre travail.

${ }^{2}$ Marie Caroline Watson Hamlin, Legends of Le Détroit. Illustrated by Miss Isabella Stewart, Détroit, Gale Research Co., 1977 [c 1883], 317 p.

${ }^{3}$ Marie Caroline Watson Hamlin, Legends of Le Détroit. Illustrated by Miss Isabella Stewart, CIHM/ICMH collection de microfiches, $\mathrm{n}^{\circ} 06685,1980$, 4 microfiches.

${ }^{4}$ Marie Caroline Watson Hamlin, Le Détroit des légendes, traduit de l'américain par Richard Ramsay, [préface de Brigitte Purkhardt], Sudbury, La Société historique du Nouvel-Ontario ( Documents historiques», $\mathrm{n}^{\circ}$ 88-89), 1991, $188 \mathrm{p}$.

${ }^{5}$ Marie Caroline Watson Hamlin, Du Crapaud à cheval au Nain rouge. Légendes de Détroit. Texte traduit et annoté par Richard Ramsay. Prologue par Brigitte 
plus récente édition que nous utilisons dans la présente étude ${ }^{6}$.

\section{II - UN ÉCRIVAIN MAL CONNU}

On connaît peu de choses sur Marie Caroline Watson Hamlin. On pourrait croire, à la lecture de son ouvrage, qu'il s'agit d'une dame d'un certain âge, qui aime à se rappeler «le bon vieux temps». Or, le "prologue» à la plus récente édition de la traduction affirme que l'auteur serait né en 1850, qu'elle aurait épousé le 13 juin 1878 un certain William Yates Hamlin (1847-1902, mercier de son état), mais que la date de sa mort demeure inconnue ${ }^{7}$. S'il est vrai qu'elle est née en 1850, elle n'avait donc que 34

Purkhardt, Sainte-Foy, Les Éditions de la Huit, 2000, 272 p. Le prologue de Brigitte Purkhardt reprend en partie, mais en l'amplifiant, sa prêface à l'édition de la Société historique du Nouvel-Ontario.

Le titre, plus accrocheur, donné à la réédition de cette traduction renvoie à deux personnages qui apparaissent dans l'œuvre, en l'occurrence, le nain rouge, considéré tout à la fois comme un ennemi irréductible des Français et de leur établissement, un messager de la mort et, pour tout dire, le «Démon de la cité des détroits» (Édition 2000, p. 50). Quant au «crapaud à cheval", il s'agit du militaire américain John Francis Hamtranck, membre du premier régiment d'infanterie des États-Unis, que, par jalousie, le chef amérindien Thayendanegea, aussi connu sous le nom de Joseph Brant, appelait ainsi parce que, comme l'explique Marie Caroline Watson Hamlin, dans une note de son ouvrage, "trapu et quelque peu voûté, [il] offrait une piètre silhouette à dos de cheval» (ibid., p. 255, note 1 renvoyant à la p. 147 du texte proprement dit).

On notera, par ailleurs, le glissement de sens intervenu, dans le choix du sous-titre donné à cette nouvelle édition de la traduction, "Légendes de Détroit », qui a le désavantage d'attirer l'attention plutôt sur la ville de Détroit, que sur toute la région du Détroit, telle qu'était l'intention du titre retenu par Marie Caroline Watson Hamlin pour son cuvre, intention que préservait le titre donné à la première édition de la traduction.

${ }^{6}$ Toutes nos citations de l'œuvre sont tirées de cette édition. Les références de page seront données entre parenthèses, directement dans le texte, en fin de citation.

${ }^{7}$ Du Crapaud à cheval au Nain rouge. Légendes de Détroit, «Prologue», p. IX, note 1 . Nous n'avons pas réussi, pour notre part, à confirmer d'une façon qui nous satisfasse les renseignements d'ordre biographique qui sont fournis dans cette note. Par ailleurs, les catalogues de bibliothèque que nous avons pu consulter, même le savant catalogue de la Bibliothèque du Congrès, à 
ans l'année où parut son livre. Il semble par ailleurs qu'elle ait été toute sa vie résidente de Détroit et qu'elle ait été à la fois férue d'histoire et fière des origines françaises de sa ville. Elle était également fascinée par les vieilles légendes, celles qui avaient une incidence locale en particulier, et par leur prolongement dans les croyances populaires. Marie Caroline Watson Hamlin tentera donc de combiner en un seul ouvrage ce double intérêt, historique d'une part, ethnologique de l'autre, ce qui explique vraisemblablement en partie la structure quelque peu hybride de son livre, avec son cadre historique qui s'étend de l'année 16691670 , moment où les missionnaires sulpiciens François Dollier de Casson et René de Bréhant de Galinée abordent pour la première fois à la terre de Détroit, à l'année 1815 , alors que suite à la guerre de 1812 , la vocation «américaine» de Détroit est désormais irrémédiablement fixée. C'est à l'intérieur de ce cadre historique qu'elle parsème et recrée, avec un à-propos qui paraît parfois un peu forcé, les légendes qui ont enchanté son imagination d'enfant et de jeune femme.

\section{III - LES INTENTIONS DE L'OUVRAGE}

Ce faisant, Marie Caroline Watson Hamlin aspire, comme elle l'affirme dans sa dédicace, à faire plaisir à de vieux amis, en particulier ceux qu'elle a fréquentés à "Tonnancour», la résidence d'été de son ami Theodore Parsons Hall, à Grosse-Pointe, où elle a entendu évoquer plusieurs de ces vieilles légendes.

Mais elle s'inscrit également dans un mouvement plus vaste qui au Québec, notamment, à la même époque, aspirait à remettre à l'honneur ces vieux récits de la tradition orale dont certains, comme l'on sait, remontent à la nuit des

Washington, semblent ignorer tout à fait les dates qui marquent le début et le terme de la vie de l'auteur. De même, des demandes de renseignements adressées à la Detroit Historical Society et à la Historical Society of Michigan sont restées sans réponse. 
temps. C'est donc tout naturellement que l'on pense, en lisant Marie Caroline Watson Hamlin, à l'injonction placée en épigraphe, en 1861, au premier numéro des Soirées canadiennes, la revue que venaient de fonder à Québec l'abbé Henri-Raymond Casgrain et ses amis: «Hâtons-nous de raconter les délicieuses histoires du peuple avant qu'il les ait oubliées", citation attribuée à Charles Nodier, comme l'on sait, mais qui, comme Réjean Robidoux l'a bien montré il y a plus de quarante ans, dans son mémoire de diplôme d'études supérieures présenté à l'Université Laval, avait été traficotée (pratique dont l'abbé Casgrain avait l'habitude) afin de ne pas effaroucher les chastes esprits des lecteurs canadiens ${ }^{8}$. On sait le profit que devaient tirer de ces vieilles légendes pour leur œuvre des littérateurs tels Philippe Aubert de Gaspé, Honoré Beaugrand, Édouard Faucher de Saint-Maurice, Louis Fréchette, Joseph-Charles Taché, pour ne nommer que ceux-là.

On imagine facilement que le recueil de Marie Caroline Watson Hamlin a dû s'élaborer lentement - probablement sur un certain nombre d'années, au gré de ses lectures - et de ses recherches - en histoire et en folklore, au gré, aussi, des récits qui lui étaient rapportés. En témoigne, à notre avis, la facture même de l'ouvrage - 31 courts récits (ou esquisses) de trois ou quatre pages en moyenne, tentant chaque fois une réconciliation - parfois malaisée - entre un événement historique et la légende à raconter. C'est ainsi que défilent progressivement sous les yeux du lecteur tous les temps forts de l'histoire du Détroit - depuis le passage

\footnotetext{
${ }^{8}$ La citation intégrale, tirée de la Légende de Sour Béatrix, se lisait ainsi: «Hâtons-nous d'écouter les délicieuses histoires du peuple avant qu'il les ait oubliées, avant qu'il en ait rougi, et que sa chaste poésie, honteuse d'être nue, se soit couverte d'un voile comme Ève exilée du paradis" (le souligné est de nous) (Charles Nodier, Légende de Sour Béatrix, Paris, Maurice Glomeau, 1924, p. 13. Voir Réjean Robidoux, "Les Soirées canadiennes et le Foyer canadien dans le mouvement littéraire québécois de 1860 ", dans Fonder une littérature nationale. Notes d'histoire littéraire, Ottawa, Éditions David, 1994, p. 39).
} 
des premiers missionnaires venus du cœur de la Nouvelle France, en passant par la fondation du fort Pontchartrain par Cadillac en 1701, par les premiers affrontements avec la puissance britannique à peine quelques années plus tard vers 1712-1713, par la chute de la Nouvelle France et ses répercussions autour des dates fatidiques de 1760 et 1763 , pour aboutir enfin au passage de la ville de Détroit dans le giron américain en 1796 et à la fixation de son destin à la fin de la guerre de 1812 .

\section{IV - UN UNIVERS EN fluX, MAIS UNE CIVILISATION DÉJÀ BIEN CARACTÉRISÉE}

C'est dire que l'on est, tout au long de l'ouvrage, dans un univers en flux, rempli d'incertitudes. Sous le régime français, les commandants de ce fort lointain de l'empire français d'Amérique se succèdent à vive allure. Après 1763 , la population est assujettie à tous les contrecoups qui vont agiter pendant plus de cinquante ans une bonne partie du continent nord-américain. Autour de ces événements et conséquences historiques ne s'en constitue pas moins une civilisation, française d'origine et d'inspiration, tout à la fois semblable à celle de l'épicentre de la Nouvelle France et différente. Cette civilisation s'inscrit dans une configuration géographique particulière (les lacs Sainte-Claire et Érié, la rivière Détroit), elle possède ses lieux mythiques chargés avec les ans de significations historiques particulières (Belle-Isle, Grosse-Pointe, Windmill Point, Presque Isle, Connor's Creek, le ruisseau Parent, devenu par la suite «Bloddy Run», etc.). Mais surtout, elle possède ses "[é]tranges histoires, singulières coutumes et admirables traditions - héritage sacré qui fut préservé d'une génération à l'autre", comme le dit Marie Caroline Watson Hamlin dans son "Introduction" (p. 1).

C'est de cette dernière matière que l'auteur fera son miel. Se déploiera alors, à travers les divers récits, comme l'a fait observer Jean-Pierre Pichette dans son relevé eth- 
nologique de l'œuvre ${ }^{9}$, toute la panoplie des lieux hantés, des revenants, des prédictions de l'avenir, des malédictions, des esprits maléfiques (le «lutin», les feux follets, le loup-garou), la chasse-galerie ${ }^{10}$ avec, en plus, une exploration de coutumes observées dans la région mais qui n'ont rien d'ésotérique, sinon à l'occasion, dans l'une ou l'autre de leurs variantes locales, pour le lecteur familier du genre: la plantation du mai, les crêpes du mardi gras, le pain bénit, le feu de la Saint-Jean, la guignolée et la galette des rois.

Souvent, c'est l'auteur qui évoque quelque souvenir lointain logé au fond de sa mémoire: «Dans la pénombre de ma mémoire vagabonde le souvenir indistinct d'une légende associée à l'endroit", écrit-elle par exemple au récit XXIII (p. 169). D'autres fois, elle fait appel à un conteur ou une conteuse - , qui se trouve comme par hasard présent ou présente à quelque rencontre conviviale, et dont Marie Caroline Watson Hamlin fait semblant de rapporter le récit, mais dans des mots qui n'en sont pas moins les siens.

Seule frustration pour le lecteur, et encore plus pour le chercheur, et constante encore, celle des sources de l'auteur, sur lesquelles elle est peu loquace: à peine, dans son "Introduction", une kyrielle de noms d'historiens (Parkman et Rameau de Saint-Père, entre autres) et de ce que l'on appellerait maintenant des ethnologues primitifs (Charlevoix, La Hontan), qu'elle remercie de l'aide qu'ils lui ont apportée à «bien servir la justesse des événements historiques» (p. 1).

${ }^{9}$ Jean-Pierre Pichette, Répertoire ethnologique de l'Ontario français, Ottawa, Presses de l'Université d'Ottawa, 1992, p. 138.

${ }^{10}$ Dans son ouvrage La chasse-galerie, de la légende au mythe (Montréal, XYZ, coll. «Théorie et littérature», 1992), Brigitte Purkhardt, après Jean Du Berger (voir p. 54), affirme que le récit de Marie Caroline Watson Hamlin, rédigé en 1883 et qu'elle retient pour sa part au nombre de quatre récits «exemplaires" auquel son étude s'intéresse (p.9), constitue "[l]e plus ancien conte du corpus des récits de chasse-galerie» (p. 129). 


\section{V - UN MONDE IDÉAL, CONVENU}

Sur un plan plus proprement littéraire, soit celui de l'écriture, on est plongé, ici, dans un monde idéal, que vient idéaliser plus encore l'ambiance légendaire des différents récits. Dans ce monde idéal, la nature est invariablement luxuriante quand elle est favorable, mais exagérément déchaînée et néfaste quand elle ne l'est pas. Les jeunes filles, qu'elles soient d'origine française ou amérindienne, sont toutes d'une beauté exquise et les jeunes officiers portent invariablement beau dans leurs uniformes rutilants. Les guerriers amérindiens, de leur côté, sont d'une droiture et d'une bravoure exemplaires dès lors qu'ils sont des alliés des Français, mais d'une férocité et d'une cruauté extraordinaires quand ils pactisent avec l'ennemi. Les êtres maléfiques ont, bien entendu, le cour entièrement noir. Bref, on navigue dans un monde convenu, simple malgré la complexité historique, un monde aux couleurs de l'univers légendaire, axé sur de violents contrastes.

En fait, l'ouvrage entier, pour peu que l'on y regarde, repose sur toute une série de tensions ou d'oppositions: celles, d'une part, des missionnaires (représentants de la "civilisation») et des Amérindiens, à qui les premiers cherchent à imposer des coutumes et des valeurs différentes de celles de leurs croyances traditionnelles; celles des missionnaires, encore, et des autorités civiles autour, notamment, de la question de l'eau-de-vie, qui revient sans cesse comme une sorte de refrain (ou serait-ce l'une des obsessions de l'auteur?); celles qui, dans la trame historique de l'ouvrage, de façon croissante, opposent la France et l'Angleterre; celles des tribus amérindiennes entre elles, selon qu'elles sont alliées des Français ou des Anglais; celles, enfin, comme cela va de soi dans un ouvrage de ce genre, entre la religion et les croyances populaires. 
Les rapports entre les individus sont élaborés sur le même modèle: plusieurs récits présentent des couples de personnages (mari et femme, amoureux et amoureuse, frère et sœur), dont l'un des membres (la femme, généralement - mais l'on voit également, au sein d'une même famille, par exemple, des frères qui s'opposent sur ce plan, ou encore deux femmes qui ont, sur ces questions, des vues divergentes), dont l'un des deux a plus tendance que l'autre à la superstition ou à la crédulité, mais qui l'emporte souvent sur ce dernier après avoir prouvé, feu follet, ou loupgarou ou fantôme ou avatar du diable à l'appui, que sa croyance n'était pas sans "fondement". De même, les amours sont généralement contrariées ou impossibles: jeune Française qui s'éprend d'un bel officier anglais ou d'un Amérindien, ou vice-versa, situations qui se résolvent habituellement par un drame ou un sacrifice (mort de l'une des parties, entrée au couvent, etc.).

\section{VI - LA PRÉSENCE AUCTORIALE}

À travers toutes les légendes et les péripéties qu'elle raconte, Marie Caroline Watson Hamlin ne renonce pas entièrement, pour autant, à sa propre présence auctoriale. Elle porte à l'occasion son regard intérieur au-delà de ce qu'elle raconte et s'interroge alors, par une réflexion morale, sur les tenants et aboutissants de l'état d'esprit qui préside à la vie de bien de ses personnages. Ainsi, écritelle,

[d]e nature, les attitudes de l'homme réfléchissent les diverses variations qui se produisent dans son environnement. Peut-on se surprendre que celui qui grandit en territoires mi-sauvages puisse découvrir cette lueur de surnaturel qui se cache au cour de la forêt vierge, se marie aux mythes indiens et rejoint la superstition latente en chacun - lueur teintant d'une signification occulte et mystique le moindre désordre ou événement singulier de la nature? (p. 100) 
Et elle a aussi, parfois, des opinions personnelles bien tranchées, tel ce cri du cœur que lui arrache, près de cinquante ans après le fait, le vandalisme perpétré dans l'île aux Bois-Blancs à l'occasion des événements de 1837, événements qui, en principe, ne la concernaient guère, elle, citoyenne américaine. Le vandalisme dont il est question consistait en l'abattage d'une magnifique forêt de tilleuls d'Amérique, véritable "couronne de gloire" de cette île, comme elle la décrit, pour la remplacer par un poste d'observation militaire, en ce "court moment de panique à caractère patriotique que des soi-disant "patriotes" baptisèrent du nom de "guerre des Patriotes de 1837" " (p. 79). C'est là le seul événement historique auquel Marie Caroline Watson Hamlin fait allusion en dehors des balises temporelles qu'elle avait données à son ouvrage.

\section{Conclusion}

Que dire maintenant, en conclusion à ce bref tour d'horizon littéraire du Détroit des légendes, sinon que s'y profile, tout au long, le vieux rêve nostalgique d'une Amérique française, rêve qui, au moment où Marie Caroline Watson Hamlin écrit son ouvrage, n'était sans doute plus guère qu'une chimère, dans une ville comme Détroit qui, tout au long du XIX ${ }^{e}$ siècle, avait subi de plein fouet les assauts de la révolution industrielle à saveur américaine. Tels sont les effets des frontières politiques que les guerres ou les révolutions viennent inopinément dresser entre les êtres. C'est là, hélas, la rançon de l'histoire. Mais survivent pourtant toujours, dans l'esprit de quelques-uns, des souvenirs, des récits qui ne cessent d'enchanter leur imagination - et celle de leurs lecteurs.

Resterait évidemment à faire une étude des aspects historiques et ethnologiques de l'œuvre, mais cette tâche dépasse nettement nos compétences. 